\title{
The double macchiato years; awards for the best basic science and epidemiology papers in 2012
}

\author{
Paul Cullinan, ${ }^{1}$ Clare Lloyd ${ }^{2}$
}

${ }^{1}$ Department of Occupational and Environmental Medicine, Imperial College (NHLI), London, UK

2Department of Lung Biology, Imperial College (NHLI), London, UK

\section{Correspondence to}

Prof Paul Cullinan,

Department of Occupational and Environmental Medicine, Imperial College London, South Kensington Campus, London SW7 2AZ, UK; p.cullinan@imperial.ac.uk

Accepted 31 May 2013
To cite: Cullinan P, Lloyd C. Thorax 2013;68:777-779.

\begin{abstract}
It's increasingly difficult to get published in Thorax so we commend all those who managed it in 2012; and salute all those who tried and failed. We think that comparisons are invidious but our chief editors, with all their schoolboy charm, disagree so here are our awards for the best basic science and epidemiological manuscripts in the year of the London Olympics.
\end{abstract}

\section{BASIC SCIENCE}

Articles in the Basic Science section have spanned all of the sections in Thorax, from asthma to chronic obstructive pulmonary disease (COPD) via respiratory infections, pulmonary fibrosis, acute lung injury and cough. Contributions have used in vitro cell culture systems to interrogate disease pathway biology, in vivo models to assess role of molecules in disease pathophysiology and patient biopsies to correlate expression of molecules and clinical phenotypes.

A gratifying number of studies used primary airway cells even though these are significantly more challenging than growing transformed cell lines. This was exhibited in a study by Fonceca et $a l^{1}$ that showed a marked difference in the response to respiratory syncytial virus in primary airway cells isolated from children compared with cell lines available commercially. Similar methods were used by Vareille et $a l^{2}$ who showed that antiviral immunity to rhinovirus is impaired in patients with cystic fibrosis (June issue). Airway cells from patients with cystic fibrosis had increased viral load in conjunction with a significantly reduced interferon response compared with cells from controls. Addition of type I and type III interferons was able rescue this response.

Asthma was the most predominant disease investigated in manuscripts under the basic science umbrella. A number of studies tackled the role of infection as a complication in the development of disease pathology. The gold medal in this section goes to a study by Essilfie who examined the molecular mechanisms behind bacterial persistence in allergic airways disease. ${ }^{3}$ A combination of inhaled house dust mite in conjunction with Haemophilus influenza infection led to neutrophilic allergic airways disease and development of a Th17 response, both of which were resistant to treatment with steroids. The data suggest that targeted treatment of bacterial infections during steroid resistant asthma may be beneficial. The challenge will be to determine more specifically which molecules involved in antibacterial immunity are defective in the asthmatic lung so that more tailored immune therapy can be developed, rather than just giving more antibiotics. Mechanisms underlying allergic sensitisation were investigated by Post et al, by comparing commercially available extracts of house dust mite. ${ }^{4}$ Interestingly, although the capacity of house dust mite to alter epithelial barrier function and immune responses were related to allergic sensitisation when tested in an in vivo mouse model, the effects were independent of serine or cysteine protease activity. This is important since the protease function of allergens has been proposed as a potential avenue for therapeutic intervention. This study highlights the importance of epithelial barrier function and development of immune sensitisation.

The potential for stem cells as therapy for respiratory diseases was highlighted in the June issue by two papers that used mesenchymal stem cells to modulate disease progression in vivo. Curley et al determined that transfer of mesenchymal stem cells (MSCs) improved recovery from ventilator induced injury and furthermore tissue repair processes were enhanced by increasing production of the anti-inflammatory cytokine IL-10, while downregulating alveolar tumour necrosis factor (TNF) levels. ${ }^{5}$ Similarly administration of MSCs to mice with $E$ coli-induced pneumonia resulted in enhanced survival and bacterial clearance. In part this effect was due to the increase in levels of the antimicrobial peptide lipocalin 2 in the airway lumen. ${ }^{6}$ Although different disease models, these studies showed that delivery of MSCs during disease promotes the paracrine secretion of molecules that promote resolution of inflammation. These studies are awarded a joint silver medal for their investigations into a whole new therapeutic area and highlighting the potential of stem cells as a therapeutic avenue for human lung disease, as eloquently discussed in the accompanying editorial ${ }^{7}$ Although animal model studies are highly suggestive that MSCs have potential as immunomodulators in a number of different pulmonary diseases, controlled trials in human subjects are urgently needed.

Mechanisms of injury in COPD were investigated in a number of different systems. Increased elastin degradation in the skin was observed in patients with COPD and was related to severity of emphysema. ${ }^{8}$ This was attributed to an imbalance in proteolytic activity, particularly in the matrix metalloproteinase family. Autoimmunity to elastin has also been proposed as a mechanism behind disease progression in COPD but the idea is controversial due to conflicting studies. A manuscript in the August issue set out to investigate $\mathrm{T}$ cell and $\mathrm{B}$ cell responses to elastin in peripheral blood samples from a large well characterised cohort of patients with COPD. ${ }^{9}$ Antielastin antibodies were measured 
in all subjects and elastin-specific T cell responses in a subgroup. Interestingly, although there was no significant antielastin antibody response in patients with CPOD, T cell immunity against collagen $\mathrm{V}$ was increased in a subgroup of smokers with COPD. These data don't entirely rule out a role for elastin autoimmunity in COPD, since the authors acknowledge that their study did not take into account antigen specificity and clonal expansion of $\mathrm{B}$ cells and $\mathrm{T}$ cells in the lung. It seems that more detail immunoanalysis of cells from patients with COPD, ideally from the lung itself, are needed before controversy regarding role of autoimmune mechanisms in COPD can be settled.

TNF family members are thought to drive inflammation in a number of pulmonary conditions. In a report in the March issue a novel reagent was used to specifically target the p55 subunit of the TNF receptor during ventilator induced lung injury. ${ }^{10}$ Domain antibodies are made from the variable domains of immunoglobulin light or heavy chains and are thus the smallest functional antigen binding portions of the IgG molecule. Their advantage is that they do not induce Fc effector responses and their small size promotes more effective delivery. Administration of a domain antibody specific for the p55 subunit of the TNF receptor was found to attenuate inflammation and respiratory function in mice following ventilator induced lung injury. The selective specificity of the domain antibody allowed functions of the p55 subunit to be modulated without affecting function of the p75 subunit, and outlined a role for p55 in neutrophil recruitment, chemokine generation and deterioration in respiratory mechanics that are indicative of ventilator induced lung injury. The role of another TNF family member, tumour necrosis factor-related apoptosis-inducing ligand (TRAIL) was investigated during pulmonary fibrosis using TRAIL deficient mice. ${ }^{11}$ TRAIL is important for regulation of cell survival and is in clinical trials for cancer. McGrath et al presented data to show that mice lacking functional TRAIL had significantly worse lung inflammation and fibrosis in a bleomycin model of lung fibrosis. Moreover, patients with idiopathic pulmonary fibrosis (IPF) showed lower levels of TRAIL than healthy controls, implying that TRAIL therapy may be of benefit in IPF and raises the possibility of using TRAIL as a potential biomarker for IPF. This study wins our bronze medal for study combining for mouse modelling with confirmation in clinically relevant samples.

\section{EPIDEMIOLOGY}

Under 'epidemiology' we considered traditional populationbased research manuscripts and those that covered public and occupational health. We had a good number of submissions of original studies in occupational lung diseases, an area we are keen to support especially when the work is from rapidly industrialising parts of the world. A fine example of such was Wang et al's 36 year follow-up of almost 600 workers in an asbestos textile factory in China. ${ }^{12}$ Exposed only to chrysotile, mined elsewhere in the same country and allegedly free from any amphibole, they nonetheless had a strikingly high rate of deaths from lung cancer. The study was not unflawed (and we found it difficult to get non-partisan reviews for this manuscript) but its findings should be a stark reminder, if one was needed, that the 'magic' mineral-banned in over 50 countries but still used in very large quantities in India, China, Russia, Kazakhstan and Indonesia among others-cannot reasonably be used 'safely' and that a very large number of people are going to die from working with it. This is a topic that crosses over from medicine to big business (if you ever have an idle moment, check out the website of the 'Chrysotile Institute') to geopolitics; we are proud to award this paper our 'gold medal' for epidemiology in
2012. In the same month we published an analysis of 11 pooled case-control studies of lung cancer which included over 13000 cases of the disease. ${ }^{13}$ Peters et al reported a small increase in risk among those with occupational exposure to organic dust (but not endotoxin or contact with animals); this is mildly controversial since earlier studies have suggested that some such groups-notably dairy farmers in Italy-have a reduced risk of lung cancer. Finally, in October we were pleased to publish the first UK study of occupational exposures and COPD. ${ }^{14}$ In around 2000 men living in Sheffield, a city with a proud legacy of heavy industry, there was an association between COPD, measured with spirometry, and a self-report of past occupational exposure to respiratory irritants; but not, interestingly, when such exposure was measured using a job-exposure matrix, a supposedly more objective assessment. This is an area fraught with potential confounding (see for example ${ }^{15}$ ) and we look forward to studies aimed at discovering how far the reported associations are genuinely causal.

Is indoor air the new outdoor air? Annesi-Maesano et al think so. In August, timed nicely for les grand vacances and while Paris sulked, we published their report of poor air quality in 100 French primary schools. ${ }^{16}$ High levels of formaldehyde, acrolein and particulates were associated with allergic asthma and rhinoconjunctivits in pupils. We are pleased to award this work our epidemiological silver medal; the study made big waves in the French media and who are we to disagree? Meanwhile, at the other end of life-and of the world-70 elderly Australians with asthma spent a winter keeping a symptom diary and making measurements of their lung function. Franklin et al suggested that exposure to unflued gas heaters-an important source of domestic pollution in many homes-gave rise to wheeze and small decrements in peak expiratory flow/forced expiratory volume in one second $\left(\mathrm{FEV}_{1}\right) \cdot{ }^{17}$ Indoor air is probably a major influence on respiratory symptoms and function in older people and we need to understand it better.

May was the lung cancer edition of Thorax. Concerns that the British public doesn't understand lung cancer (or don't want to understand it) were reflected by two papers. A group from London developed and tested a 'lung cancer awareness measure' and concluded that the UK population has a low awareness of lung cancer symptoms and risk factors and that any efforts to encourage earlier presentation will have to remedy this. ${ }^{18}$ In a similar vein Patel et al, in an interesting qualitative study, identified four types among those who chose not to take part in a screening programme: 'too old to be bothered', 'worriers', 'fatalists' and 'avoiders'. ${ }^{19}$ We plan to use all or some of these terms at our next meeting with our editorial chiefs. Last, but certainly not least, we published the findings of a trial of an intervention designed to advance lung cancer diagnosis in primary care. ${ }^{20}$ In six areas of northern England with high rates of lung cancer, a marketing campaign seemed to increase the rates of chest X-ray requests in primary care and lung cancer diagnoses, although the latter rise was not statistically significant. This is difficult work but we like publishing trials and would have been pleased to award this one our bronze medal if we hadn't been pipped to the post by its award in the 'clinical' category. ${ }^{21}$

We know we ought to but we don't especially enjoy debates about how lung function should be measured. However two studies published in August did impress us and we are going to award them joint bronze medals. Both concerned bronchodilator responsiveness. In 4000 'healthy' non-smokers taking part in the worldwide Burden of Obstructive Lung Disease survey the pooled estimate of the upper $95 \%$ confidence limit for $\mathrm{FEV}_{1}$ response was $280 \mathrm{mls}$ or $12 \%$ from baseline. ${ }^{22}$ We thought we 
knew this but now we know it for sure. To shake us from complacency, however, findings from the Evaluation of COPD Longitudinally to Identify Predictive Surrogate Endpoints study $^{23}$ suggest that bronchodilator responsiveness in COPD is temporally unstable, no different from that in disease-free smokers and a poor predictor of mortality and morbidity. An accompanying editorial ${ }^{24}$ advised caution: '... we would propose that bronchodilator testing is interpreted with caution ... because a large proportion of patients with asthma and COPD will have values within the healthy range'.

So, congratulations to all those mentioned above and to the rest, please remember the Baron's words: 'the most important thing in the Olympic Games is not winning but taking part; the essential thing in life is not conquering but fighting well'.

Contributors $\mathrm{PC}$ and $\mathrm{CL}$ wrote this jointly.

Competing interests None.

Provenance and peer review Commissioned; internally peer reviewed.

\section{REFERENCES}

1 Fonceca AM, Flanagan BF, Trinick $R$, et al. Primary airway epithelial cultures from children are highly permissive to respiratory syncytial virus infection. Thorax 2012;67:42-8.

2 Vareille M, Kieninger E, Alves MP, et al. Impaired type I and type III interferon induction and rhinovirus control in human cystic fibrosis airway epithelial cells. Thorax 2012;67:517-25.

3 Essilfie AT, Simpson JL, Dunkley ML, et al. Combined Haemophilus influenzae respiratory infection and allergic airways disease drives chronic infection and features of neutrophilic asthma. Thorax 2012;67:588-99.

4 Post S, Nawijn MC, Hackett TL, et al. The composition of house dust mite is critical for mucosal barrier dysfunction and allergic sensitisation. Thorax 2012;67:488-95.

5 Curley GF, Hayes M, Ansari B, et al. Mesenchymal stem cells enhance recovery and repair following ventilator-induced lung injury in the rat. Thorax 2012;67:496-501.

6 Gupta N, Krasnodembskaya A, Kapetanaki M, et al. Mesenchymal stem cells enhance survival and bacterial clearance in murine Escherichia coli pneumonia. Thorax 2012;67:533-9.

7 Mac Sweeney R, McAuley DF. Mesenchymal stem cell therapy in acute lung injury: is it time for a clinical trial? Thorax 2012:67:475-6.
8 Maclay JD, McAllister DA, Rabinovich R, et al. Systemic elastin degradation in chronic obstructive pulmonary disease. Thorax 2012;67:606-12.

9 Rinaldi M, Lehouck A, Heulens $N$, et al. Antielastin B-cell and T-cell immunity in patients with chronic obstructive pulmonary disease. Thorax 2012; 67:694-700.

10 Bertok S, Wilson MR, Morley PJ, et al. Selective inhibition of intra-alveolar p55 TNF receptor attenuates ventilator-induced lung injury. Thorax 2012;67:244-51.

11 McGrath EE, Lawrie A, Marriott HM, et al. Deficiency of tumour necrosis factor-related apoptosis-inducing ligand exacerbates lung injury and fibrosis. Thorax 2012:67:796-803.

12 Wang $X$, Yano E, Qiu H, et al. A 37-year observation of mortality in Chinese chrysotile asbestos workers. Thorax 2012:67:106-10.

13 Peters $\mathrm{S}$, Kromhout $\mathrm{H}$, Olsson $\mathrm{AC}$, et al. Occupational exposure to organic dust increases lung cancer risk in the general population. Thorax 2012;67:111-16.

14 Darby AC, Waterhouse JC, Stevens V, et al. Chronic obstructive pulmonary disease among residents of an historically industrialised area. Thorax 2012;67:901-7.

15 Ramsay SE, Whincup PH, Lennon LT, et al. Longitudinal associations of socioeconomic position in childhood and adulthood with decline in lung function over 20 years: results from a population-based cohort of British men. Thorax 2011;66:1058-64.

16 Annesi-Maesano I, Hulin M, Lavaud F, et al. Poor air quality in classrooms related to asthma and rhinitis in primary schoolchildren of the French 6 Cities Study. Thorax 2012;67:682-8

17 Franklin PJ, Loveday J, Cook A. Unflued gas heaters and respiratory symptoms in older people with asthma. Thorax 2012;67:315-20.

18 Simon AE, Juszczyk D, Smyth $N$, et al. Knowledge of lung cancer symptoms and risk factors in the U.K.: development of a measure and results from a population-based survey. Thorax 2012;67:426-32.

19 Patel D, Akporobaro A, Chinyanganya N, et al. Attitudes to participation in a lung cancer screening trial: a qualitative study. Thorax 2012;67:418-25.

20 Athey VL, Suckling RJ, Tod AM, et al. Early diagnosis of lung cancer: evaluation of a community-based social marketing intervention. Thorax 2012;67:412-17.

21 Bush A, Pavord I. Thorax: the Cappuccino years. Thorax 2013:68:1-4.

22 Tan WC, Vollmer WM, Lamprecht B, et al. Worldwide patterns of bronchodilator responsiveness: results from the Burden of Obstructive Lung Disease study. Thorax 2012;67:718-26

23 Albert $\mathrm{P}$, Agusti $\mathrm{A}$, Edwards $\mathrm{L}$, et al. Bronchodilator responsiveness as a phenotypic characteristic of established chronic obstructive pulmonary disease. Thorax 2012;67:701-8

24 Fingleton J, Weatherall M, Beasley R. Bronchodilator responsiveness: interpret with caution. Thorax 2012;67:667-8. 